\section{Vaccine Effectiveness against DS-1-Like Rotavirus Strains}

Toyoko Nakagomi ${ }^{1}$

Author affiliation: Retired from Nagasaki University, Nagasaki, Japan

DOI: https://doi.org/10.3201/eid2601.191377

To the Editor: Global emergence of reassortant rotavirus A (RVA) strains possessing the DS-1 backbone genes, such as DS-1-like G1P[8] strains (1-3), raises 2 key questions of public health importance. First, is the G1P[8] monovalent vaccine (RV1) effective against the DS-1-like G1P[8] strain? Second, does this strain cause more severe disease than the Wa-like G1P[8] strain?

For the first question, Jere et al. (1) showed that RV1 was highly effective against DS-1-like G1P[8] RVAs (vaccine effectiveness [VE] 85.6\% [95\% CI $34.4 \%-96.8 \%])$. For the second question, we conducted a study in Hanoi, Vietnam, during the 2012-2013 rotavirus season in which 20 DS-1-like G1P[8] RVAs co-circulated with 49 Wa-like G1P[8] RVAs and 50 G2P[4] RVAs among children $<2$ years of age who had diarrhea. We found no evidence of increased virulence of DS-1-like G1P[8] strains as measured by Vesikari's severity scores (3).

However, Jere et al. (1) were unable to demonstrate statistically significant VE against DS-1-like G2P[4] or Wa-like G1P [8] strains and were concerned about the low point estimate of the VE against heterotypic G2P[4] strains. Using the previous dataset (3), we conducted a post hoc logistic regression analysis to compare any RV1 vaccination status versus 0 -dose RV1 vaccination status between strain-specific rotavi-

\footnotetext{
${ }^{1}$ Retired.
}

rus diarrhea case-patients and 127 acute respiratory infection control patients whose RV1 coverage was 49.6\%. We calculated VE against DS-1-like G1P[8] as $72.5 \%$ (95\% CI 10.8\%-91.5\%; $\mathrm{p}=0.045)$, VE against Wa-like G1P[8] as 90.8\% (95\% CI 72.9\%-96.9\%), and VE against G2P[4] as $78.1 \%$ (95\% CI $49.1 \%-90.6 \%$ ). These findings confirm the effectiveness of RV1 against fully heterotypic G2P[4] strains, as shown elsewhere $(4,5)$, and supplement the study by Jere et al. (1) in helping dismiss concern about continued use of the monovalent vaccine, even in places where RVAs with the DS-1 backbone are not uncommon.

\section{References}

1. Jere KC, Bar-Zeev N, Chande A, Bennett A, Pollock L, Sanchez-Lopez PF, et al. Vaccine effectiveness against DS-1-like rotavirus strains in infants with acute gastroenteritis, Malawi, 2013-2015. Emerg Infect Dis. 2019;25:1734-7. https://doi.org/10.3201/eid2509.190258

2. Fujii $Y$, Nakagomi T, Nishimura N, Noguchi A, Miura S, Ito $\mathrm{H}$, et al. Spread and predominance in Japan of novel G1P[8] double-reassortant rotavirus strains possessing a DS-1-like genotype constellation typical of G2P[4] strains. Infect Genet Evol. 2014;28:426-33. https://doi.org/10.1016/ j.meegid.2014.08.001

3. Nakagomi T, Nguyen MQ, Gauchan P, Agbemabiese CA, Kaneko M, Do LP, et al. Evolution of DS-1-like G1P[8] doublegene reassortant rotavirus A strains causing gastroenteritis in children in Vietnam in 2012/2013. Arch Virol. 2017;162:739-48. https://doi.org/10.1007/s00705-016-3155-6

4. Correia JB, Patel MM, Nakagomi O, Montenegro FM, Germano EM, Correia NB, et al. Effectiveness of monovalent rotavirus vaccine (Rotarix) against severe diarrhea caused by serotypically unrelated G2P [4] strains in Brazil. J Infect Dis. 2010;201:363-9. https:/ / doi.org/10.1086/649843

5. Braeckman T, Van Herck K, Meyer N, Pirçon JY, Soriano-Gabarró M, Heylen E, et al.; RotaBel Study Group. Effectiveness of rotavirus vaccination in prevention of hospital admissions for rotavirus gastroenteritis among young children in Belgium: case-control study. BMJ. 2012;345:e4752. https://doi.org/10.1136/ bmj.e4752

Address for correspondence: Toyoko Nakagomi, 14-1-604, Ohashi, Nagasaki 852-8134, Japan; email: tnakagom@gmail.com 